\title{
Practice and Effect of Specialized High Quality Nursing Care in Operating Room
}

\author{
Ke Lin, Hongwei Cai ${ }^{*}$, Jing Bai, Siyu Liu, Qi Fan, Qintai Zhang, Li Tang \\ Department of Operating Room, the Second Affiliated Hospital of Kunming Medical University, Kunming, China
}

Email address:

77340786@qq.com.(Hongwei Cai)

*Corresponding author

\section{To cite this article:}

Ke Lin, Hongwei Cai, Jing Bai, Siyu Liu, Qi Fan, Qintai Zhang, Li Tang. Practice and Effect of Specialized High Quality Nursing Care in Operating Room. Clinical Medicine Research. Vol. 8, No. 5, 2019, pp. 125-130. doi: 10.11648/j.cmr.20190805.16

Received: September 16, 2019; Accepted: October 4, 2019; Published: October 23, 2019

\begin{abstract}
This study aimed to explore the practical effect and reasonable evaluation methods of specialized high quality nursing care in operating room. Stratified random sampling was used to select 93 nurses from the operating rooms of two affiliated hospitals of Kunming Medical University in Yunnan Province. A set of "Specialized High Quality Nursing Care Items in Operating Room" was implemented in the operating rooms of the two hospitals simultaneously. The "Task Performance and Contextual Performance Scale" was used to measure and collect data of nurses in operating room before and after the implementation of the items. After the "Specialized High Quality Nursing Care Items in Operating Room" was implemented, the task performance total score and dimension scores of patient care, nursing information provision and specialized nursing skills were (58.59 \pm 1.77$),(19.27 \pm 1.16)$, (19.50 \pm 1.11$),(19.80 \pm 0.70)$, respectively, higher than the respective scores $(37.21 \pm 1.92),(5.55 \pm 0.70),(13.78 \pm 1.12),(17.87 \pm 0.98)$ before the implementation, and the differences were statistically significant $(\mathrm{t}=-82.48$ to $-15.15, \mathrm{P}<0.01)$. After the "Specialized High Quality Nursing Care Items in Operating Room" was implemented, the contextual performance total score and dimension scores of mutual support among colleagues, extra burden outside work tasks, and support to the organization were (70.43 \pm 2.94$)$, (28.23 \pm 1.21$),(18.52 \pm 1.29),(23.67 \pm 1.86)$, respectively, higher than the respective scores $(36.20 \pm 1.79),(15.52 \pm 1.94),(7.33 \pm 1.11)$, $(13.34 \pm 0.87)$ before the implementation, and the differences were statistically significant $(\mathrm{t}=-91.99$ to $-46.68, \mathrm{P}<0.01)$. In conclusion, the "Specialized High Quality Nursing Care Items in Operating Room" is a series of nursing care that can improve task performance and contextual performance of nurses in operating room and promote the quality of high-quality nursing in operating room. The task performance and contextual performance can also comprehensively and effectively evaluate the implementation effect of specialized high quality nursing care in operating room.
\end{abstract}

Keywords: Operating Room, Nursing Care, Task Performance, Contextual Performance

\section{Introduction}

In the "Notice on Conducting Evaluation of High Quality Nursing Care", the National Health and Family Planning Commission required hospitals at all levels in China to conduct a three-year (2014-2017) evaluation of the effect of high quality nursing care, including high quality nursing care conducted in outpatient (emergency) clinics and operating rooms [1], aiming to analyze the implementation effect of high quality nursing care through effective evaluation mechanism, constantly promote high quality nursing care, and improve the quality of clinical nursing care. Operating room nurses are the main body of high quality nursing care implementation, the implementation of their clinical nursing work directly affects and reflects the quality of specialized nursing care and the effect of perioperative treatment $[2,3]$, and is an important indicator to effectively measure the quality of specialized high quality nursing.

In this study, a set of specialized high quality nursing care items was implemented in the operating rooms of two large comprehensive hospitals from March to December 2016, and the "task performance" and "contextual performance" of specialized nurses in operating rooms were measured before and after the implementation to judge the effect of specialized high quality nursing care items in operating room on the quality of specialized nursing of operating room nurses. At the same time, it is inferred that "task performance" and 
"contextual performance" are a set of methods that can effectively evaluate and comprehensively reflect the quality of specialized high quality nursing in operating room.

\section{Subjects and Methods}

\subsection{Subjects}

This study is a prospective study carried out in the operating rooms of two affiliated hospitals of Kunming Medical University, which are both Class A tertiary hospitals in Yunnan Province. The number of beds, the number of patients in and out of hospital, hardware facilities, the difficulty of surgical operations carried out by hospitals, the types of operations, the annual volume of operations, the number of operating rooms, and the human resources situation are basically the same. The allocation of human and material resources in operating room has a certain consistency, with no significant structural and physical differences. Stratified random sampling was used to select the nurses in operating rooms of the two hospitals. Inclusion criteria were: (1) nurses with $\geq 1$ year specialized clinical experience in operating room; (2) nurses with Certificate of Nursing Practice, People's Republic of China; (3) participants' informed consent to the study and voluntary participation. Exclusion criteria were: (1) new nurses; (2) nurses in standardized training. According to Yamane formula, the sample size of this study was calculated and determined $[4] . \mathrm{n}=\mathrm{N} /\left[1+\mathrm{N}\left(\mathrm{e}^{2}\right)\right] ; \mathrm{N}=$ the total number of samples that meet the criteria; $\mathrm{n}=$ sample size; $\mathrm{e}=$ sample error, defined as 5\%. The total number of nurses selected in operating rooms of the two hospitals in this study is 102 , calculated according to the formula, $\mathrm{n}=102 \div\left(1+102 \times 0.05^{2}\right)=$ 81 . To ensure the validity of sample collection, Best and Kahn [5] suggested that $20 \%$ of the sample should be added to the sample size of the experiment to avoid sample loss. Therefore, the final planned sample in this study is determined to be 97 .

\subsection{Methods}

(1) Implementation steps of the study

The study was completed by five steps: 1) after contacting the nursing department and operating room of the two selected hospitals, the researchers submitted the experimental application in writing and obtained the consent of the hospitals; 2) the researchers recruited two experimental assistants in operating room of each hospital through voluntary registration to assist in completing the experiment; 3) from March to April 2016, in operating rooms of the two hospitals, the researchers completed random sampling and the first round of scale distribution and data collection, respectively; (4) From May to October 2016, the researchers simultaneously implanted a series of "Specialized High Quality Nursing Care Items in Operating Room" into the daily nursing work of operating rooms of the two hospitals. After the completion of the work every Friday, the experimental assistants regularly implemented various cares and reported in writing the implementation to the researchers, who performed analysis and dynamic adjustment to ensure the effectiveness of the implementation of all specialized high quality nursing care items; 5) From November to December 2016, the researchers performed the second round of scale distribution and collection on nurse samples that had received the first round of data collection.

(2) Implementation of specialized high quality nursing care items in operating room Since 2010, the operating room of the Second Affiliated Hospital of Kunming Medical University, guided by the goal and connotation of high quality nursing, has successively conducted and implemented a series of specialized high quality nursing care in operating room including the following contents: "focusing on surgical patients, improving the level of specialized nursing care in operating room, refining the contents of specialized nursing, deepening the professional connotation and implementing the nursing responsibility system". Through five years of exploration and practice, the operating room of the hospital has achieved remarkable results in this work, and has obtained a relatively mature implementation system and method of high quality nursing care. In this study, with the specialized high quality nursing care items that are successfully conducted in operating room of the hospital, according to the situation of the selected hospitals and the feasibility of the item implementation, seven main items of specialized high quality nursing care in operating room were formulated, and each item was completed through the specific implementation of several sub-items, which reflect specialized high quality nursing care in operating room mainly at two levels, i.e., high quality nursing care items centered on surgical patients and high quality nursing care items centered on improving the level of specialized nursing care (Table 1).

Table 1. Specialized high quality nursing care implementation items in operating room.

\begin{tabular}{|c|c|c|c|}
\hline $\begin{array}{l}\text { Care conduction } \\
\text { level }\end{array}$ & No. & $\begin{array}{l}\text { High quality nursing } \\
\text { care main items }\end{array}$ & Execution sub-items \\
\hline \multirow{2}{*}{$\begin{array}{l}\text { high quality } \\
\text { nursing care items } \\
\text { centered on } \\
\text { surgical patients }\end{array}$} & 1 & $\begin{array}{l}\text { perioperative high } \\
\text { quality health } \\
\text { education care }\end{array}$ & $\begin{array}{l}\text { Specially-assigned person completes } 100 \% \text { preoperative visiting care for surgical patients. } \\
\text { preoperative visits to issue "love card" care } \\
\text { to provide surgical information and surgical cognitive support for family members of surgical patients } \\
\text { to provide specialized and individualized care for special surgical patients } \\
\text { enhanced health education content during waiting period for patients after admission } \\
\text { postoperative high quality nursing follow-up service for surgical patients }\end{array}$ \\
\hline & 2 & $\begin{array}{l}\text { perioperative high } \\
\text { quality safety nursing } \\
\text { care }\end{array}$ & $\begin{array}{l}\text { seamless accompany nursing for surgical patients after admission } \\
\text { zero tolerance of errors in surgical safety check } \\
\text { perioperative hypothermia risk preventive nursing care for surgical patients } \\
\text { perioperative pressure ulcer risk preventive nursing care for surgical patients } \\
\text { perioperative incision infection preventive nursing care for surgical patients }\end{array}$ \\
\hline
\end{tabular}




\begin{tabular}{|c|c|c|c|}
\hline $\begin{array}{l}\text { Care conduction } \\
\text { level }\end{array}$ & No. & $\begin{array}{l}\text { High quality nursing } \\
\text { care main items }\end{array}$ & Execution sub-items \\
\hline $\begin{array}{l}\text { high quality } \\
\text { nursing care items } \\
\text { centered on } \\
\text { improving the } \\
\text { level of } \\
\text { specialized } \\
\text { nursing care }\end{array}$ & 5 & $\begin{array}{l}\text { perioperative high } \\
\text { quality sensory nursing } \\
\text { care }\end{array}$ & $\begin{array}{l}\text { zero tolerance of surgical specimen disposal errors } \\
\text { perioperative nursing standardized terminology care of operating room nurses } \\
\text { perioperative smile nursing care of operating room nurses } \\
\text { "surgical environment adaptation" nursing care for children under } 12 \text { years old } \\
\text { to provide listening to soothing music service during waiting period for patients after admission } \\
\text { to establish operating room nursing specialized group with the same specialty and uniform } \\
\text { standards according to uniform specifications, procedures and requirements } \\
\text { The specialized group draws up the specialized nursing work plan in Gantt chart form, and } \\
\text { promotes and implements specialized nursing work according to the Gantt chart plan. } \\
\text { Various specialized operating groups in operating room carry out the implementation, management, } \\
\text { progress supervision and assessment of various tasks according to the plan in PDCA mode. } \\
\text { Various specialized groups in operating room complete the evaluation of the implementation effect } \\
\text { of specialized work in } 360^{\circ} \text { evaluation form. } \\
\text { to establish and implement a surgical level and specialized nurse level matching system } \\
\text { to establish and implement a surgeon level and specialized nurse level matching system } \\
\text { to establish and implement a system for surgical incision infection and tracking implementation of } \\
\text { responsible nurses } \\
\text { to establish and implement a system for intraoperative pressure ulcer of surgical patients and } \\
\text { tracking implementation of responsible nurses } \\
\text { to establish and implement a system for surgical safety check, execution and implementation of } \\
\text { responsible nurses } \\
\text { to establish and implement a green channel for emergency surgery } \\
\text { to establish and implement a delivery and entry process for operating room items } \\
\text { to establish and implement waiting time minimization mechanism for consecutive operation } \\
\text { to establish and implement punctually cutting mechanism for selective surgery }\end{array}$ \\
\hline
\end{tabular}

\subsection{Research Tools}

(1) General questionnaire

After literature review, the general questionnaire of this study was designed according to the purpose and content of the study, including hospital, gender, age, working years, unit personnel establishment, shift, education, professional title, and marital status.

(2) Nursing task performance and contextual performance scale. The measurement scale of this study was designed and developed by Greenslade and Jimmieson [6] of Queensland University, Australia in 2007 according to Borman and Motowwidlo's two-dimensional theory of work performance [7]. It has good reliability and validity [8-10]. The internal consistency Cronbach $\alpha$ coefficient of the source scale shows that the internal consistency of all dimensions is greater than 0.7. The internal consistencies of the items of task performance and contextual relationship performance are 0.91 and 0.90 , respectively. The results of convergent validity and labeling validity are good. After obtaining the consent and authorization of the authors of the source scale, the researchers translated the scale. During the translation process, three experts with rich clinical nursing experience were invited. Meanwhile, English-proficient experts used the method of "translation-back translation" to check the consistency of language expression of the scale. The scale consists of 6 dimensions and 25 items, of which 3 dimensions are used to measure task performance: patient care, nursing information provision, specialized nursing skills; the other 3 dimensions are used to measure contextual performance: mutual support among colleagues, extra burden outside work tasks and support to the organization. Grade 1-7 scoring method is used, the higher the score, the better the work performance of nurses. The first 11 items of the scale are used to measure task performance, mainly on nurses' grade scores of differences in their daily work behavior; the item score ranges from "poor" ( 1 point) to "excellent" ( 7 points); the other 14 items are the measurement items of contextual performance, which require nurses to score the execution frequency of their different daily nursing work. The item score ranges from "never" (1 point) to "high" (7 points). The total scores of task performance and contextual performance and the measurement results of each dimension are shown by the mean of item score. Pre-test was carried out before using the scale. Among 102 nurses, the number was ranked according to the roster provided by the hospitals, one nurse was selected from every 5 nurses, 20 nurses were randomly sampled to complete the pre-test, and the obtained Cronbach $\alpha$ coefficient was 0.94 . The Cronbach $\alpha$ coefficients of task performance and contextual performance scale used in actual measurement are 0.90 and 0.93 , respectively.

\subsection{Statistical Analysis}

After the data were collected and verified, the database of Microsoft Office Excel 2003 was established for data entry, and statistical analysis was carried out by using statistical software SPSS 19.0. Descriptive analysis was performed on the general data of all descriptive subjects (age of nurses, working years, educational level, professional title, unit personnel establishment, shift situation, etc.). The change of task performance and contextual performance scores of nurses in operating room before and after the implementation of specialized high quality nursing care items was processed by paired sample $t$ test, and the statistical test level was $\mathrm{a}=0.05$. $\mathrm{P}$ 
$<0.05$ was considered statistically significant.

\section{Results}

\subsection{General Data of Operating Room Nurses}

In this study, a total of 97 questionnaires were distributed and 94 questionnaires were collected, in which one was incomplete, there were 93 valid questionnaires, and the recovery rate was $95.87 \%$. The number of nurses surveyed in operating rooms of the two hospitals was 45 (accounting for $48.38 \%$ ) and 48 (accounting for $51.62 \%$ ), respectively; the ages of 93 nurses were $(33.50 \pm 8.95)$ years, and working years were $(13.73 \pm 8.54)$ years (Table 2$)$.

Table 2. General data of 93 operating room nurses.

\begin{tabular}{lll}
\hline Items & Frequency & Composition ratio (\%) \\
\hline Age (years) & & \\
$20-30$ & 40 & 43.00 \\
$31-40$ & 29 & 31.20 \\
$41-50$ & 22 & 23.60 \\
$\geq 51$ & 2 & 2.20 \\
Length of service (years) & & \\
$1-10$ & 36 & 38.70 \\
$11-20$ & 35 & 37.60 \\
$21-30$ & 20 & 21.50 \\
$\geq 31$ & 2 & 2.20 \\
Education & & \\
master & 0 & 0 \\
undergraduate & 73 & 78.50 \\
diploma & 20 & 21.50 \\
secondary specialized school & 0 & 0 \\
Professional title & & \\
nurse & 22 & 23.70 \\
nurse practitioner & 25 & 26.80 \\
\hline
\end{tabular}

\begin{tabular}{lll}
\hline Items & Frequency & Composition ratio (\%) \\
\hline $\begin{array}{l}\text { supervisory nurse } \\
\text { deputy chief nurse } \\
\text { chief nurse }\end{array}$ & 42 & 45.20 \\
$\begin{array}{l}\text { Unit personnel establishment } \\
\text { on the staff }\end{array}$ & 4 & 4.30 \\
non-staff & 43 & 0 \\
$\begin{array}{l}\text { Shift situation } \\
\text { day shift }\end{array}$ & 50 & 46.20 \\
night shift & & 53.80 \\
Marital status & 16 & \\
unmarried & 77 & 17.20 \\
married & & 82.80 \\
divorced & 22 & 23.64 \\
\hline
\end{tabular}

3.2. Task Performance and Contextual Performance and the Implementation Effect of Specialized High Quality Nursing Care Items in Operating Room

The total score of task performance of operating room nurses was (37.21 \pm 1.92$)$ before the implementation of specialized high quality nursing care items, and the score increased to (58.59 \pm 1.77$)$ after the implementation of the items; while the total score of contextual performance was $(36.20 \pm 1.79)$ before the implementation of the items, and increased to $(70.43 \pm 2.94)$ after the implementation of the items.

For task performance score of operating room nurses, the mean differences in all dimensions were all statistically significant before and after the implementation of specialized high quality nursing care items $(\mathrm{t}=-82.48,-32.48,-15.15$, all $\mathrm{P}<0.05)$; the mean difference of contextual performance in each dimension was also significant $(\mathrm{t}=-73.03,-56.78$, -46.68 , all $\mathrm{P}<0.05$ ) (Table 3).

Table 3. Comparison of scores of each dimension of task performance and contextual performance of 93 operating room nurses before and after the implementation of specialized high quality nursing care items.

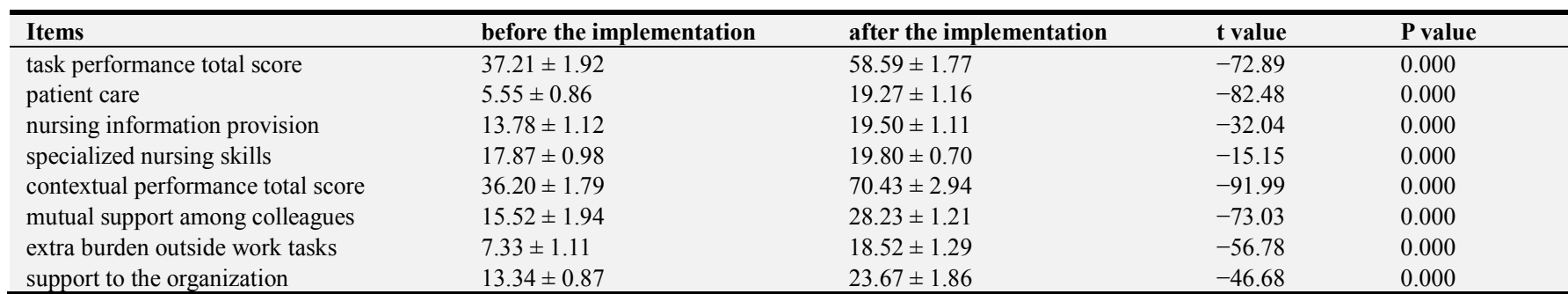

\section{Discussion}

\subsection{Significance of Task Performance and Contextual Performance Measurement of Nurses}

Clinical nursing performance measurement usually only involves the category of "task performance" related to clinical nursing skills, and mainly reflects the level of clinical nursing skills and the implementation of nursing work [11-13]. Borman and Motowidlo [7] proposed a two-dimensional classification of work performance, i.e., task performance and contextual performance. In this new theoretical model, "task performance" emphasizes skills and proficiency of work and motivation for effective completion, while "contextual performance" is a comprehensive supplement to work performance and mainly emphasizes interpersonal skills at work, creating good working relationships with partners, helping others to effectively complete team work and the motivation to support organizations. Guided by this theoretical model, Greenslade and Jimmieson designed and developed a task performance and contextual performance scale for clinical nurses to comprehensively evaluate clinical nursing performance through two dimensions of "task performance" and "contextual performance", accurately judge the level of nursing quality, and reflect the situation of clinical nursing work from multiple levels [6].

Operating room nursing is a team work, which requires 
close cooperation with specialist nurses, surgeons, anesthesiologists, pathologists, emergency department, blood transfusion department and other multidisciplinary and multidepartment medical personnel to achieve safe and orderly surgical treatment. Therefore, the two-dimensional measurement method of "task performance" and "contextual performance" is undoubtedly the best means to evaluate the work performance of nurses in operating room and the quality of specialized clinical nursing work. This study introduces the "contextual performance" dimension into the measurement of nurses' work performance, which is a comprehensive supplement to the measurement of nursing work performance. It emphasizes the interpersonal skills, coordination ability with working partners, team work ability and motivation to support organizations in the work of nurses. This feature coincides with the requirements of nursing work in operating room.

\subsection{Measurement of Task Performance and Contextual Performance of Nurses}

In this study we found that before the implementation of specialized high quality nursing care items, the task performance total score of nurses in operating room showed a medium level (37.21 \pm 1.92$)$. However, it showed a significant improvement $(58.59 \pm 1.77)$ after the implementation of the items. This change depends on the implementation effects of "perioperative high-quality health education care" and "high-quality sensory nursing care" in the items. The introduction of these items in clinical work has greatly increased preoperative communication between nurses and surgical patients, so that surgical patients can acquire sufficient surgical cognition and psychological tolerance before operation. In addition, the implementation of "standardizing care terminology", "providing smiling service", "establishing preoperative waiting for adaptation and relaxed environment" and other external sensory nursing care during perioperative period help patients adapt to the environment and obtain the best surgical response state [14]. However, in all measurement dimensions of "task performance", the "specialized nursing skills" did not show a significant improvement. This reason may be that the specialized nursing operation skills in operating room have a very rigorous and standardized inherent model in the norms and procedures, the operating room nurses must strictly abide by the norms and procedures when implementing the operation of nursing skills, and the autonomy and innovation of their operation will be affected to a certain extent. Therefore, the implementation of high quality nursing care items has little effect on "specialized nursing skills".

The scores of various dimensions of contextual performance all increased after the implementation of the items. During the implementation of the items, operating room nurses will have comprehensive understanding of their profession. This effect will promote their interaction, resonance, concern and love with the operating room nursing profession, thereby enhancing their sense of professional value and identity [15]. This explanation coincides with
Bormanan and Motowwidlo's theory that 'Employees' sense of professional value is closely related to their contextual performance [7]. The more interaction they have with their profession in work, the higher their sense of professional value, and the higher their contextual performance level. At the same time, the professional dedication spirit of employees will be greatly enhanced, which is reflected in that they will be more voluntarily engaged in work outside their duties, help other staffs in the work environment, and be willing to engage in work behavior beneficial to the organization." Among the dimensions of contextual performance, the biggest increase is "support between colleagues", and this result is closely related to the team work characteristics in operating room. The teamwork of operating room spans the whole perioperative period, from preoperative visiting, operation information checking, item counting, posture placement, intraoperative cooperation, intraoperative rescue, safety monitoring, to patients' moving and transfer, handover and follow-up and traceability after operation, all work must depend on the cooperation of a multidisciplinary medical team to be safely and effectively completed [16]. Good cohesion and effective collaboration of the operation team are the guarantee of the safety of surgical patients and quality of high quality nursing $[17,18]$.

\subsection{Implementation Effect of Specialized High Quality Nursing Items in Operating Room}

The results of this study showed that the improvement of the task performance and contextual performance of operating room nurses by specialized high quality nursing items in operating room is statistically significant $(\mathrm{P}<0.01)$. The promotion effect of specialized high quality nursing items on each dimension of task performance and contextual performance clarifies the promotion effect of the care items on specialized nursing skills, nursing safety, family care, specialist development and discipline construction in operating room. Operating room nurses can experience the positive and benevolent professional competition in specialized high quality nursing care items, and the recognition of surgical peers greatly enhances their sense of professional value and achievement. Once their work is recognized, they will gain a higher level of professional value, and their support for the organization and organizational integration will be significantly improved [19]. Therefore, the nurses in operating room show more enthusiasm for work and more supports to the organization. In addition to completing the workload, they will spend extra time to satisfy the patients and their families with special nursing needs, design and provide special perioperative nursing care, which greatly improves the quality of specialized high quality nursing care.

\section{Conclusion}

In summary, task performance and contextual performance of nurses are effective methods to reflect the quality of specialized high quality nursing care in operating room. Implementing high quality nursing care items in operating 
room could improve work performance and quality of nursing of operating room nurses, and promote the content and level of high quality nursing care in operating rooms in large general hospitals. In addition, the implementation effect of specialized high quality nursing care can be evaluated by measuring the task performance and contextual performance of nurses. In future studies, the specialized high quality nursing care in operating room should be evaluated from the viewpoint of surgical patients and surgeons to provide multi-level evidence-based support.

\section{Acknowledgements}

This work was funded by General Program of Scientific Research Fund of Yunnan Provincial Education Department (No. 2014Y175).

\section{References}

[1] National Health and Family Planning Commission of the People's Republic of China. (2014). Notice of the General Office of National Health and Family Planning Commission on Conducting Evaluation of High Quality Nursing Care. http://www.nhfpc.gov.cn/yzygj/s3593/201407/8c99ec14e65f4 289894a66c279edd08b.shtml.

[2] Knudson L, Editor M. (2014). The perioperative nurse's role in OR design. AONR J, 100 (2), C8-9.

[3] Khobreh M, Ansari F, Dornhöfer M, Vas R, \& Fathi M. (2014). Med-Assess System for Evaluating and Enhancing Nursing Job Knowledge and Performance. Open Learning and Teaching in Educational Communities, 8719, 494-497.

[4] Yamane T. (1973). Statistic: an introductory analysis. Tokyo: Harper International, 224-238.

[5] Best WJ, Kahn VJ. (2005). Research in education. 9th ed. Boston: Pearson Education, 178-183.

[6] Greenslade JH, Jimmieson NL. (2007). Distinguishing between task and contextual performance for nurses: development of a job performance scale. $J$ Adv Nurs, 58 (6), 602-611.

[7] Borman WC, Motowidlo SJ. (1993). Expanding the criterion domain to include elements of contextual performance. San Francisco: Lossey-Bass, 71-99.
[8] Ling T. Meaningful work and job performance among nurses in Harbin Medical University Hospitals, The People's Republic of China. (2014). Thailand: Faculty of Nursing, Chiang Mai University.

[9] Lin K, Liu YY, Chipakdee B, \& Chontawan R. (2012). The Relationship between Fatigue and Task Performance as well as Contextual Performance in Nurses. Journal of Kunming Medical University, 33 (8), 135-139.

[10] Greenslade HJ, Jimmieson NL. (2007). Understanding the linkages between service climate and satisfaction in a health care setting. Society of Australasian Social Psychologists Conference, Brisbane, Australia, 2007. Brtsbane: SASP, 13-15.

[11] Feddock CA, Hoellein AR, Wilson JF, Caudill TS, \& Griffith CH. (2007). Do pressure and fatigue influence resident job performance? Med Teach, 29 (5), 495-497.

[12] Wang XX, Kunaviktikul W, Thungjaroenkul P, Zheng S, \& Wang H. (2011). The status quo of job performance and its related factors among clinical nurses. Chinese Nursing Management, 10 (9), 36-38.

[13] Kong W, Zhang YQ, \& Wang L. (2015). Study on correlation between nurses' organizational trust and job performance. Chinese Nursing Research, 29 (2), 160-162, 163.

[14] Guo LH, Shi W. (2014). Scenario simulation training and effect of preoperative visits by nurses in operating room. Chin J Nurs, 49 (3), 371-373.

[15] Xiao XL, Zhang DH, Xiong LP, \& Zeng YZ. (2016). A survey study of nurses' perceived benefits and intent to stay. Chinese Nursing Management, 16 (4), 468-471.

[16] Zhang JH, Zhong Y. (2016). Effects of "Doctor-nurse Integrating" mode on the operation room nursing quality improvement. Chin J Prac Nurs, 32 (19), 1502-1504.

[17] Serino MF. (2015). Quality and patient safety teams in the perioperative setting. AORN J, 102 (6), 617-628.

[18] Zhang LQ, Zheng DH, Xu D, \& Chen LL. (2016). The effects of colleague solidarity on job satisfaction among operating room nurses. Chin J Nurs, 51 (1), 70-74.

[19] Liao X, Luo BR, Luo WY, \& Tang YX. (2016). The correlation between operating room nurses' cognition of patient safety culture and hospitals responsibility. Chin J Prac Nurs, 32 (20), 1589-1592. 